\title{
Biosynthesis of Galactofuranose in Kinetoplastids: Novel Therapeutic Targets for Treating Leishmaniasis and Chagas' Disease
}

\author{
Michelle Oppenheimer, ${ }^{1}$ Ana L. Valenciano, ${ }^{1,2}$ and Pablo Sobrado ${ }^{1,2}$ \\ ${ }^{1}$ Department of Biochemistry, Virginia Tech, Blacksburg, VA 24061, USA \\ ${ }^{2}$ Instituto Tecnológico de Costa Rica, Cartago, Costa Rica \\ Correspondence should be addressed to Pablo Sobrado, psobrado@vt.edu
}

Received 15 December 2010; Revised 2 March 2011; Accepted 14 March 2011

Academic Editor: Ariel M. Silber

Copyright (C) 2011 Michelle Oppenheimer et al. This is an open access article distributed under the Creative Commons Attribution License, which permits unrestricted use, distribution, and reproduction in any medium, provided the original work is properly cited.

Cell surface proteins of parasites play a role in pathogenesis by modulating mammalian cell recognition and cell adhesion during infection. $\beta$-Galactofuranose (Galf) is an important component of glycoproteins and glycolipids found on the cell surface of Leishmania spp. and Trypanosoma cruzi. $\beta$-Galf-containing glycans have been shown to be important in parasite-cell interaction and protection against oxidative stress. Here, we discuss the role of $\beta$-Galf in pathogenesis and recent studies on the Galfbiosynthetic enzymes: UDP-galactose 4' epimerase (GalE), UDP-galactopyranose mutase (UGM), and UDP-galactofuranosyl transferase (GalfT). The central role in Galf formation, its unique chemical mechanism, and the absence of a homologous enzyme in humans identify UGM as the most attractive drug target in the $\beta$-Galf-biosynthetic pathway in protozoan parasites.

\section{Galactofuranose}

$\beta$-Galactofuranose $(\beta$-Galf $)$ is the five-member ring isomer of galactose (Figure 1). This rare sugar was initially found in several human bacterial pathogens including Mycobacterium tuberculosis, Escherichia coli, Salmonella typhimurium, and Klebsiella pneumoniae [1-4]. In M. tuberculosis, $\beta$-Galf is found in the arabinogalactan layer where it links the peptidoglycan and mycolic acid layers [1]. In E. coli and K. pneumoniae, it is present in the $\mathrm{O}$ antigen, while in S. typhimurium it is found in the T antigen [2-4]. In all of these organisms, the enzyme UDP-galactopyranose mutase (UGM) serves as the sole biosynthetic source of $\beta$-Galf $f$ as it is responsible for converting UDP-Galp to UDP-Gal $f$ (Figures 2 and 3) [5-10]. UDP-Galf $f$ serves as the precursor molecule of $\beta$-Galf, which is attached to the various components of the cell surface by galactofuranosyl transferases (GalfTs) (Figure 2) [11, 12]. UGMs and GalfTs are not found in humans, therefore, they have been examined as potential drug targets.

Deletion of the genes coding for UGM or GalfTs has shown that these proteins are essential in M. tuberculosis, highlighting the importance for Galf in bacteria [13]. Studies have also been conducted to identify inhibitors for M. tuberculosis UGM [14-17]. These studies showed that specific inhibitors of $M$. tuberculosis UGM were able to prevent mycobacterium growth and, therefore, validated Galf biosynthesis as a drug target against mycobacteria [14].

$\beta$-Galf has also been shown to be present in fungi [18-21]. In the human pathogen Aspergillus fumigatus, it is found in four components of the cell wall: galactomannan, glycoprotein oligosaccharides, glycophosphoinositol (GPI) anchored lipophosphogalactomannan (LPGM), and sphingolipids $[18,22]$. Deletion of the UGM and the Galf transporter genes in Aspergillus resulted in attenuated virulence, increased temperature sensitivity, and thinner cell walls $[23,24]$. Gal $f$ is also present in protozoan parasites and is a virulence factor [25]. In Leishmania spp., it is present in the lipophosphoglycan (LPG) and in glycoinositolphospholipids (GIPLs). In T. cruzi, Galf is found in the GIPLs and glycoprotein oligosaccharides [26, 27]. This paper focuses on current knowledge on the biosynthetic pathway of $\beta$-Galf 
and its role in the pathogenesis of T. cruzi and Leishmania spp.

1.1. Overview of T. cruzi and Leishmania spp. T. cruzi is the causative agent of Chagas' disease, which often develops severe cardiac complications in patients with the chronic form of the disease [28]. In the T. cruzi life cycle, the parasite undergoes three developmental stages as it is transmitted from the insect vector (triatomine bug) to mammals: trypomastigote (vector feces and mammalian bloodstream), epimastigote (vector midgut), and amastigote (mammalian smooth muscle) [29]. Leishmania spp. are the causative agents of leishmaniasis, which can manifest in three formsvisceral, cutaneous, or mucocutaneous-depending on the species [30]. In the Leishmania spp. lifecycle, there are two stages: the amastigote (mammalian host macrophages) and the promastigote stage (vector (sand fly) midgut) [30].

Current treatments are limited due to toxic side effects and cost, therefore new drugs are needed [31-33]. Lifecycle progression of both T. cruzi and Leishmania spp. is associated with changes in the carbohydrate composition on the cell surface. These changes are important for mediating host-pathogen interactions Gal $f$ levels and Galf-containing glycans are shown to be modulated throughout the parasite life cycles and are important for pathogenesis [26, 34-36]. As Galf biosynthesis has been shown to be an attractive drug target for other pathogens, enzymes involved in this pathway may also prove to be ideal drug targets for the treatment of Chagas' disease and leishmaniasis.

\section{Biosynthesis of Galf in Kinetoplastids}

The biosynthesis of Galf begins with the uptake and metabolism of galactose $(\mathrm{Gal})$. Gal is an epimer of glucose that differs only by the orientation of the hydroxyl group at the carbon 4 position. Gal is a component of lactose in milk, is present in grains and beets, and can be utilized for energy after conversion to glucose (Glc). Gal is also a major component of glycans, present in proteins and lipids in most organisms, ranging from bacteria to mammals. The metabolism of Gal occurs via the Isselbacher or Leloir pathways (Figure 2). In the Leloir pathway, Gal is converted to glucose-6-phosphate (Glc-6-P), an intermediate in glycolysis (Figure 2(a)). After Gal is transported into the cytoplasm by hexose transporters it is phosphorylated by galactokinase (GalK). Phosphorylation of Gal prevents its transport out of the cell. Gal-1-phosphate (Gal-1-P) is then coupled to uridyl diphosphate by galactose-1-phosphate uridyltransferase (GalPUT) yielding two products, UDPGal and Glc-1-phosphate (Glc-1-P). UDP-Gal is converted to UDP-glucose (UDP-Glc) by UDP-glucose-4-epimerase (GalE). Glc-1-P is isomerized to Glc-6-P by phosphoglucomutase (PGM) [37, 38]. In the Isselbacher pathway, Gal-1-P can be directly converted to UDP-Gal by the enzyme UDPsugar-pyrophosphorylase (USP) (Figure 2(b)) [39]. These pathways contribute to the pool of UDP-Gal required for the biosynthesis of the glycocalyx.

In Leishmania spp., galactose has been shown to be obtained from the environment by hexose transporters

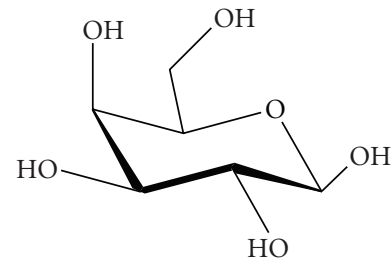

$\beta$-galactopyranose

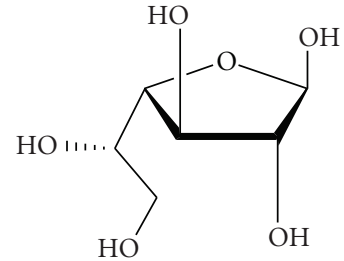

$\beta$-galactofuranose
FIGURE 1: Structures of $\beta$-Galactopyranose and $\beta$-Galactofuranose.

through radioactive labeling assays, and both the Leloir and Isselbacher pathways function to maintain proper levels of UDP-Gal [40]. The Isselbacher pathway is present in $L$. major due to the wide substrate specificity of USP, which can convert many sugars to the corresponding UDP-sugar including glucose, galactose, galacturonic acid, and arabinose [41]. The wide range of substrate specificity has been explored by crystallographic studies and has been attributed to a larger active site that can alter conformations of residues involved with sugar binding and the flexibility of the sugarbinding loop [42]. Deletion of the USP gene in L. major showed that the protein is nonessential and demonstrates that since the Leloir and Isselbacher pathways are redundant, proteins involved with the formation of UDP-Gal are not essential for Leishmania spp. survival $[41,43]$. In T. cruzi and Trypanosoma brucei, galactose cannot be obtained from the environment because it is not recognized by the hexose transporters; therefore, these parasites rely on the action of GalE from the Leloir pathway for the direct conversion of UDP-Glc to UDP-Gal for galactose [37, 44, 45]. In both $T$. cruzi and L. major, UDP-Gal is converted to UDP-Galf by UGM (Figures 2(c) and 3) [7]. UDP-Galf is the substrate for several UDP-galactofuranosyl transferases, which decorate many glycoproteins and glycolipids on the cell surface of $T$. cruzi and L. major.

2.1. Galactofuranose-Containing Proteins and Lipids. Galf is found in many major components of the glycocalyx of Leishmania spp. and T. cruzi. In Leishmania spp., and Galf is found in the lipophosphoglycan (LPG) and in glycoinositolphospholipids (GIPLs), while in T. cruzi, Galf is found in the GIPLs and glycoprotein oligosaccharides (Figure 4) $[26,27]$. In this section, we will describe the structure and role in pathogenesis of known Galf-containing glycoconjugates.

2.1.1. Lipophosphoglycan (LPG) from Leishmania. LPG from Leishmania spp. has four components: a phosphoinositol lipid, a core oligosaccharide, phosphoglycan (PG) repeat units, and a cap (Figure $4(\mathrm{a})$ ). $\beta$-Gal $f$ is found in the core structure where it plays a role in connecting the PG repeat units to the phospholipid [35, 46]. LPG has been found to be important for adhesion to the sandfly midgut, resistance to the human complement $\mathrm{C} 3 \mathrm{~b}$, protection from oxidative stress, and prevention from phagosomal transient fusion [47-50]. 


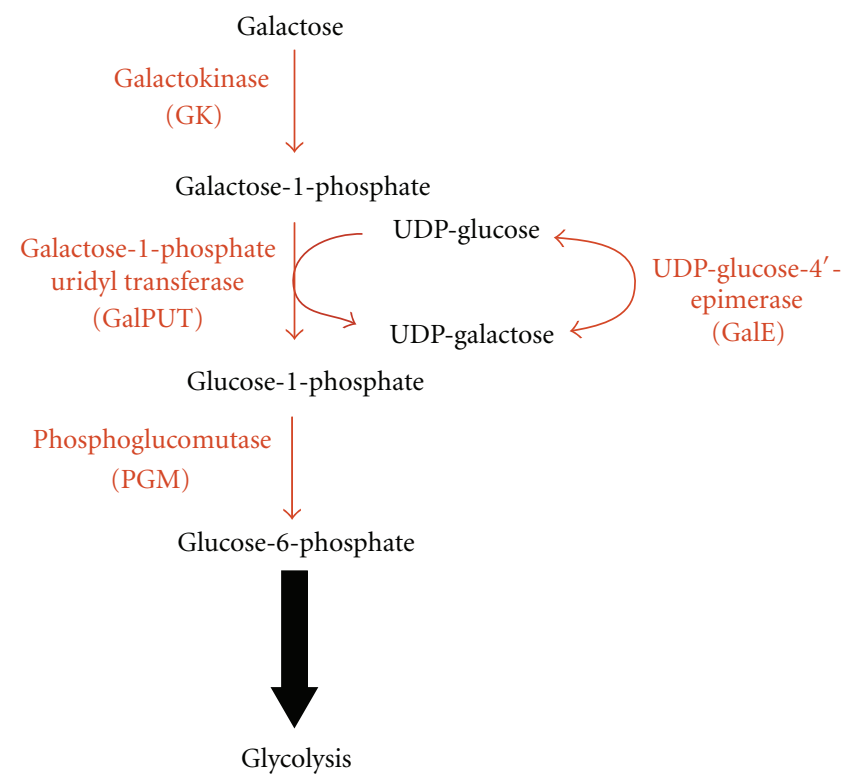

(a)

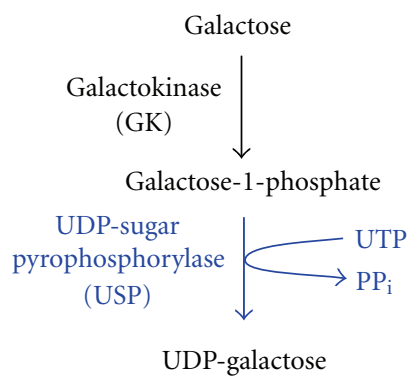

(b)

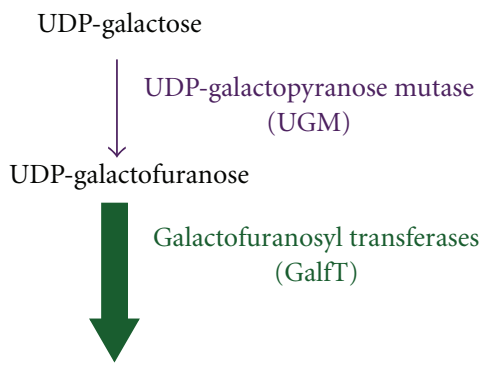

Glycocalyx

Figure 2: Biosynthetic pathways of Galf. (a) In the Leloir pathway, Gal is transported to the cytoplasm where it is converted to galactose-1phosphate by galactokinase (GK). Galactose-1-phosphate uridyl transferase (GalPUT) and UDP-Glc 4' epimerase (GalE) are involved in the synthesis of UDP-galactose. (b) Alternatively, galactose can be directly converted to UDP-galactose by the Isselbacher pathway by UDP-sugar pyrophosphorylase (USP). (c) UDP-Galactose is then converted to UDP-Galf by UDP-galactopyranose mutase (UGM), and UDP-Galf is subsequently added to the glycocalyx by Galactofuranosyl transferases (GalfT).

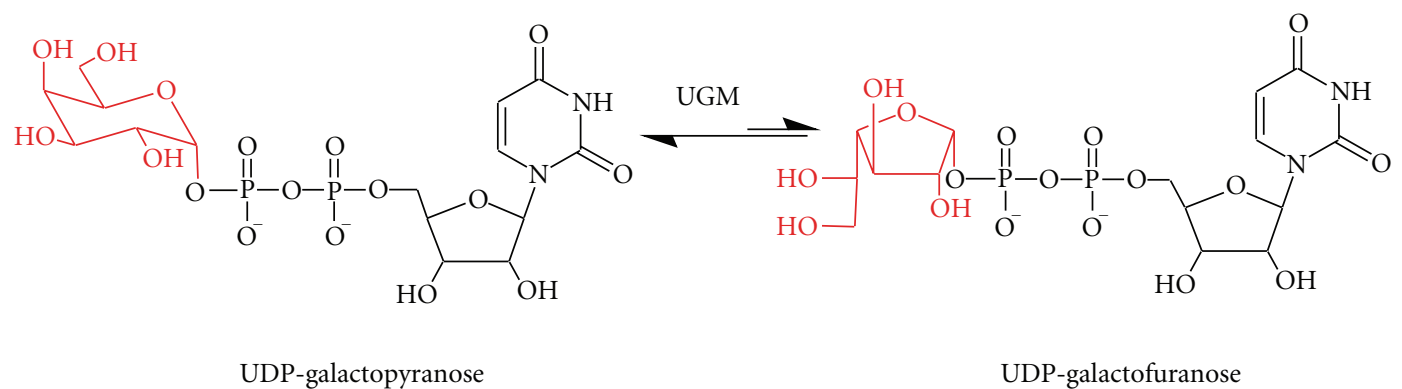

FIGURE 3: Reaction catalyzed by UDP-Galactopyranose mutase (UGM).

2.1.2. Glycoinositolphospholipids (GIPLs). GIPLs are free glycosylated phospholipids found in many kinetoplastids. Those found in Leishmania spp. and T. cruzi are considered unique due to the presence of $\beta$-Galf (Figures 4(b) and 4(c)) $[26,51-54]$. GIPL structure is species and strain dependent and varies in expression levels throughout the life stages of the parasite [55-58]. GIPLs from Leishmania spp. are thought to be precursor molecules for the synthesis of the LPG core structure [59]. L. major GIPL-1 has been shown to be involved in parasite-host interactions and is thought to play an important role in establishing infection $[57,60]$.

GIPLs from T. cruzi include a class of phospholipids previously identified as lipopeptidophosphoglycans (LPPGs) [61-63]. The LPPGs were originally considered a separate class from the GIPLs due to the presence of contaminating amino acids during their purification; these amino acids have since been identified as part of the NETNES [27, 64]. The importance of GIPLs in T. cruzi is revealed by studies that show that it plays a role in antigenicity, both with rabbit and human sera $[36,53]$. The antigenicity is thought to be primarily due to the terminal $\beta$-Gal $f$ residues either from the GIPLs or the O-linked mucins, as removal of $\beta$-Galf results in decreased levels of antigenicity $[36,53,65]$. It has also been shown that GIPLs play a role in attachment of the parasite to the luminal midgut of the vector Rhodnius prolixus [55]. T. cruzi modulates this interaction by altering GIPL expression levels during its life cycle, as epimastigotes have much higher expression of GIPLs than trypomastigotes $[55,65,66]$. 


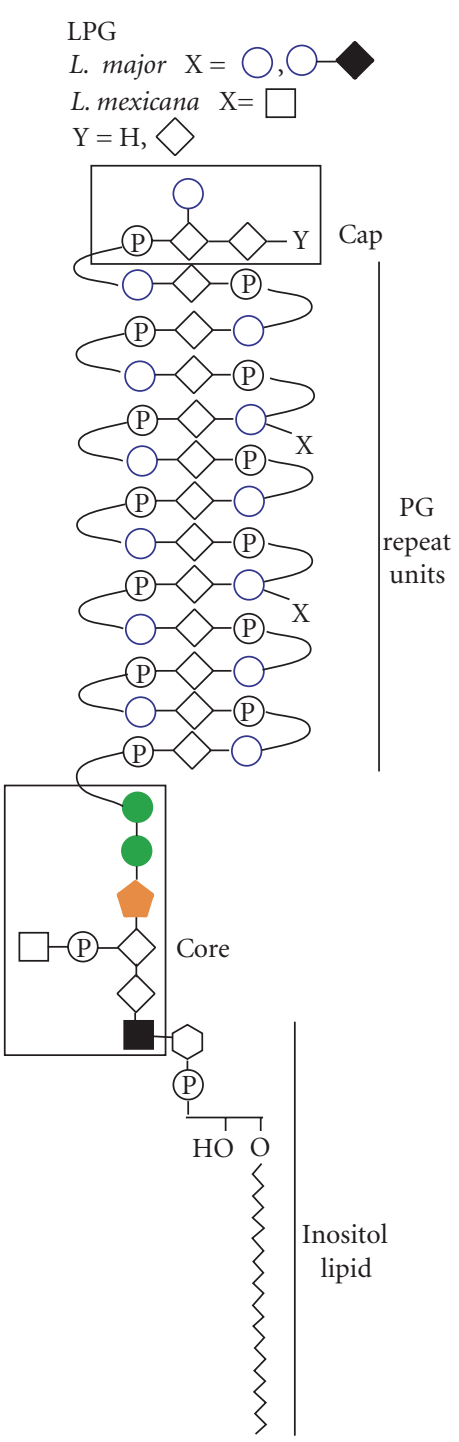

(a)

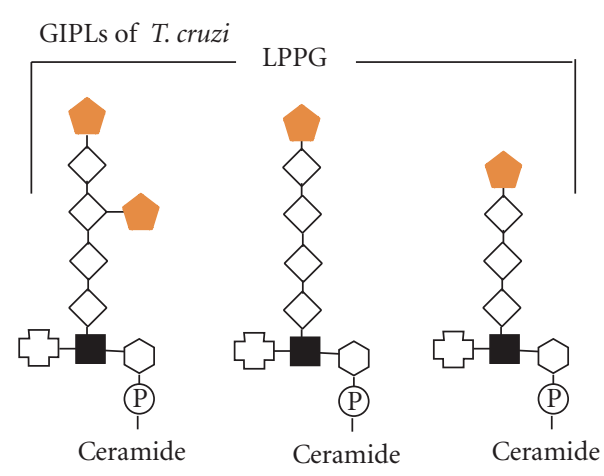

(b)

GIPL-1-3, A

L. major $\mathrm{Z}=\mathrm{H}(1), \mathrm{O}(2), \bigcirc(3), \bigcirc-O(\mathrm{~A})$
L. mexicana $\mathrm{Z}=\mathrm{O}(2),-(3)$

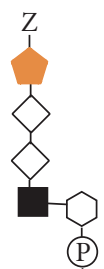

(P)

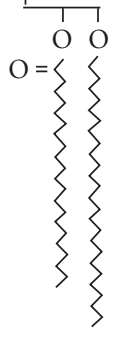

(c)

(d)

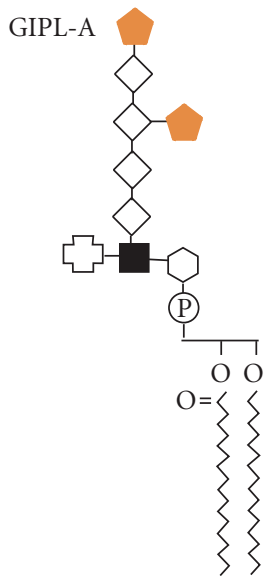

O-linked glycans

T. cruzi strains

G and Tulahuen
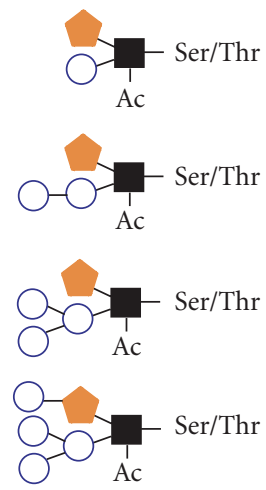

$$
\begin{aligned}
& \text { (P) }=\text { Phosphate } \\
& \bigcirc=\beta \text {-galactopyranose } \\
& =\alpha \text {-galactopyranose } \\
& \bigcirc=\text { myoinositol } \\
& \square=\alpha \text {-glucose }
\end{aligned}
$$

Figure 4: Structures of Galf-containing glycans of Leishmania spp. and T. cruzi. (a) Structure of LPG from Leishmania spp. (b) Structures of GIPLs from T. cruzi, including the previously annotated LPPG and GIPL-A (c) Structures of GIPL-1-3, (A) from L. major and L. mexicana. (d) Selected subset of structures of O-linked glycans found in both T. cruzi strains G and Tuhulan.

2.1.3. N-Linked Glycans. $\beta$-Galf is found in mannose $\mathrm{N}$ linked oligosaccharides in several species of trypanosomatid flagellates including T. cruzi, Leptomonas samueli, Herpetomonas samuelpessoai, Crithidia fasciculate, and Crithidia harmosa $[36,67-70]$. The glycan structures have been solved for L. samueli, C. fasciculate, and C. harmosa and are shown to be species dependent $[67,69]$. $\beta$-Gal $f$ units are found as terminal sugars linked to mannose residues in high mannose type $\mathrm{N}$-linked glycans $[67,69]$. The role of $\mathrm{N}$-linked glycans has currently not been significantly explored for T. cruzi. 
2.1.4. T. cruzi O-Linked Glycans and Mucins. T. cruzi mucins are a family of GPI-linked glycoproteins with high levels of O-linked glycosylation [71]. Several studies have been conducted to determine the composition of the oligosaccharides bound to Thr and Ser residues in these glycoproteins [72-76]. In T. cruzi, the O-glycans are not linked to $\mathrm{N}$ acetylgalactosamine as in mammals and other organisms; instead, they are linked to $\mathrm{N}$-acetylglucosamine [77]. It has been demonstrated that these glycans vary highly among T. cruzi strains, and $\beta$-Galf is a component of the glycan structures of $T$. cruzi strains G, Tulahuen, and Dm28c; however, $\beta$-Galf is not found in T. cruzi strains CL-Brener and Y (Figure 4(e)) [72-74, 78, 79]. These mucins play an important role in parasite-host interaction by both protecting against host defense mechanisms and ensuring targeting of specific cells and tissues [71, 77].

\section{Galactofuranose Is a Virulence Factor in Kinetoplastids}

It has been shown that incubation of L. major or T. cruzi with Galf-specific antibodies blocks parasite binding to macrophages or mammalian cells, resulting in a $50-80 \%$ decrease in infection rates $[60,66,80,81]$. It was further shown that the antibody specifically bound to the $\beta$-Galf present in GIPLs of T. cruzi and GIPL-1 of L. major [60, 66]. This suggests that $\beta$-Galf and the GIPLs of T. cruzi and GIPL-1 of L. major play a role in cell adhesion and infection. Furthermore, it was shown that macrophages incubated with p-nitrophenol- $\beta$-Galf were infected $80 \%$ less by L. major, while macrophages incubated with $p$-nitrophenol- $\beta$-Gal $p$ saw no decrease in infectivity [60]. Together, these results confirm that $\beta$-Galf plays an important role in parasite-host interaction and suggest that $\beta$-Gal $f$ biosynthetic enzymes are potential drug targets.

3.1. UDP-Glucose $4^{\prime}$-Epimerase (GalE). In T. cruzi, GalE is the first protein required for Galf biosynthesis [82]. GalE is classified as a short-chain dehydrogenase/reductase (SDR) with a conserved Tyr-X-X-X-Lys motif and a characteristic Rossmann fold structure for $\mathrm{NAD}(\mathrm{P})^{+}$binding [38, 83]. GalE is a homodimer that consists of two domains, an N-terminal domain with the Rossmann fold and a C-terminal domain that binds the substrate, UDP-Glc $[84,85]$. The catalytic site is located in the cleft between the two domains [84, 85]. The mechanism is shown to be conserved across species and involves the deprotonation of the Glc O4' hydroxyl and hydride transfer from the $\mathrm{C} 4$ carbon of Gal to NAD ${ }^{+}$ $[84,85]$. The intermediate 4-keto sugar rotates in the active site and NADH transfers back the hydride to the opposite face forming UDP-Gal $[84,85]$.

Mutant strains of T. brucei and T. cruzi with deletion of the galE gene have not been obtained suggesting that Gal metabolism is essential for parasite survival $[45,82$, $86,87]$. Conditional null mutants were created in T. brucei using tetracycline-regulated expression $[45,86]$. Studies with this strain showed that removal of tetracycline from the trypomastigote parasite led to cell death and decreased Gal surface-expression levels by $30 \%[45,86]$. These studies showed that, upon Gal starvation, Gal was eliminated from T. brucei variant surface glycoprotein (VSG) and from poly$\mathrm{N}$-acetyllactosamine-containing glycoproteins causing cell growth to cease and differentiation to a stumpy-like form, ultimately leading to cell death [87].

Single galE knockout mutants of T. cruzi epimastigotes were also constructed [82]. These cell strains showed several phenotypic differences including shortened flagella and agglutination, which is thought to be the result of a lack of surface mucins [82]. Interestingly, these cell strains show a preference for expressing high levels of Galf-containing GIPLs over Galp mucins, whose expression levels were reduced 6-9-fold, suggesting levels of Galf is preferentially maintained in the glycocalyx over Galp [82]. In Leishmania spp., Gal can be obtained from extracellular sources, presumably by a family of hexose transporters [40, 88]. Thus, GalE is not essential in these parasites.

Studies have been undertaken to identify novel inhibitors that specifically target the GalE of T. brucei $[89,90]$. Using high-throughput screens and computer modeling experiments, inhibitors that showed preference to T. brucei GalE over human GalE were identified [89, 90]. However, when these compounds were tested in vitro with T. brucei and either mammalian CHO cells or liver (MRC5) cells, these compounds either were cytotoxic to both the parasite and mammalian cells or the compound was ineffective against T. brucei $[89,90]$. These studies suggest that, while GalE remains a potential drug target, there will be many difficulties in designing specific inhibitors for the treatment of these diseases without unwanted side effects.

3.2. UDP-Galactopyranose Mutase (UGM). UGM is a flavodependent enzyme that catalyzes the conversion of UDPGalp to UDP-Galf. UGM was first identified in Escherichia coli K-12 in 1996, and since then it has been identified in several other pathogenic microorganisms including $M$. tuberculosis, L. major, T. cruzi, and A. fumigatus [5-8]. Interestingly, while $T$. cruzi produces UGM the related $T$. brucei does not, and as a result, T. brucei does not produce Galf [70]. UGM has been found to be the sole biosynthetic source of Galf and since it is not found in mammals is considered an ideal drug target.

Deletion of the UGM gene in L. major shows that this enzyme plays an important role in pathogenesis [25]. In the absence of UGM, L. major mutants were completely depleted of Galf, lacked LPG PG repeats, and contained truncated forms of GIPLs [25]. Furthermore, mice infection by L. major lacking Galf was significantly attenuated [25]. As previously mentioned, deletion of UGM also showed that Galf is a virulence factor in A. fumigatus and Aspergillus nidulans $[23,95]$. These studies show the importance of UGM and validate this enzyme as a drug target in protozoan and other eukaryotic human pathogens.

Although the reaction catalyzed by UGM does not involve a net redox change for the conversion of UDP-Galp to UDP-Galf, the reaction requires the flavin cofactor to be in the reduced form $[96,97]$. Structural and mechanistic studies of the prokaryotic UGM have led to two proposals for the ring contraction mechanism (Figure 5). One mechanism 
TABLE 1: UDP-galactopyranose mutases.

\begin{tabular}{lcccc}
\hline Species & Amino acids & \% identity & Oligomeric state & Reference \\
\hline E. coli & 367 & 100 & Dimer & Dimer \\
M. tuberculosis & 399 & 44 & Monomer & [91] \\
L. major & 491 & 15 & Monomer & b \\
T. cruzi & 480 & 15 & Tetramer & b \\
A. fumigatus & 510 & 14 & & {$[93]$} \\
\hline
\end{tabular}

a Identity to the E. coli enzyme.

${ }^{\mathrm{b}}$ Oppenheimer and Sobrado unpublished results.

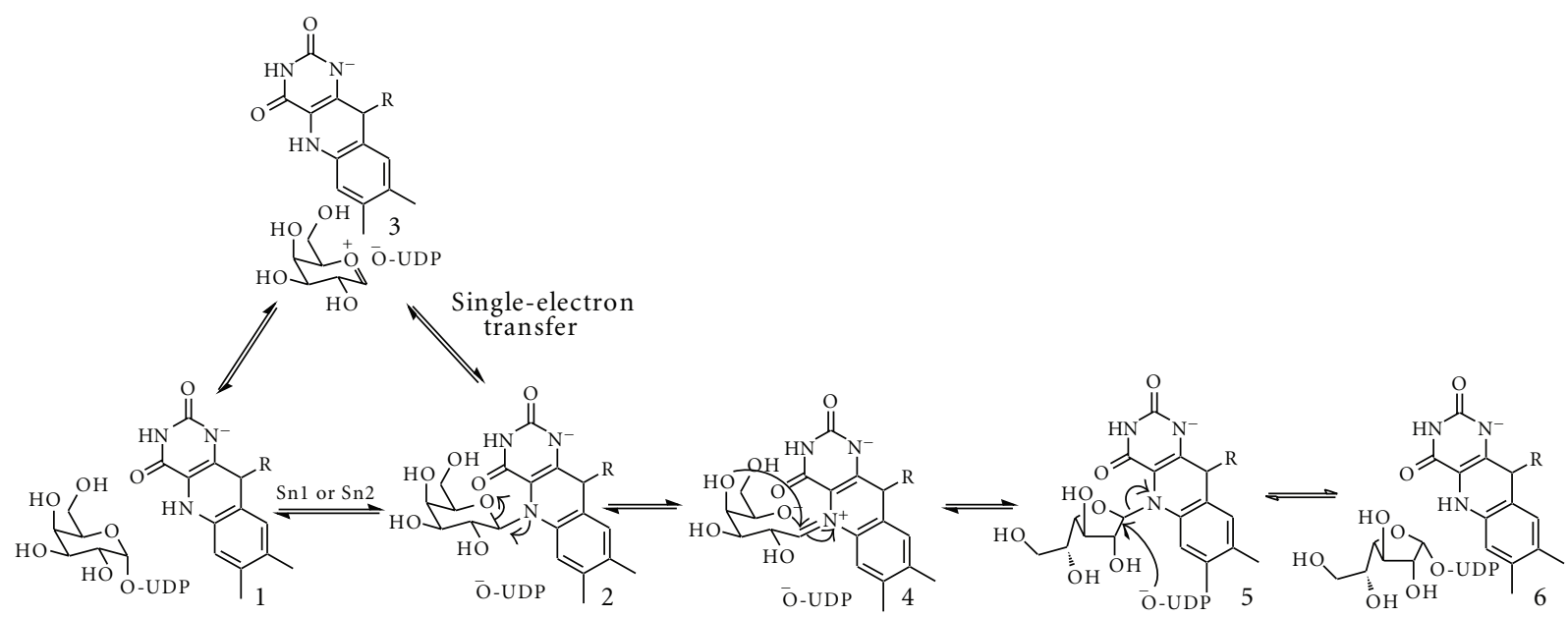

FIGURE 5: Proposed chemical mechanism for UGMs. Nucleophilic attack by the reduced flavin (1) leads to a flavin-galactose adduct (2). This step can either occur via an Sn1 or Sn2 reaction. Alternatively, the flavin can transfer one electron to a galactose oxocarbenium ion, forming a sugar and flavin radical that can also form the flavin-galactose adduct. Formation of a flavin iminium ion leads to sugar ring opening (4). Sugar ring contraction occurs by attack of the C4 hydroxyl to the C1-carbon (5). The final step is the bond formation to UDP (6).

depicts the reduced flavin acting as a nucleophile, attacking the anomeric carbon (C1) of Gal to form a flavin N5-C1 Gal adduct [98]. This adduct has been isolated and characterized in the prokaryotic UGM from K. pneumoniae [98, 99]. The other proposed mechanism involves a single-electron transfer from the reduced flavin to Gal, which then forms the sugar-flavin adduct [100].

Several structures have been solved for prokaryotic UGMs, in both oxidized and reduced states with and without substrate bound, providing excellent groundwork for the development of specific inhibitors $[92,99,101]$. The structure of prokaryotic UGMs show that it is a homodimer and a mixed $\alpha / \beta$ class protein with 3 domains: an FADbinding domain with a typical Rossmann fold, a 5-helix bundle, and a 6 -stranded antiparallel $\beta$-sheet $[91,101]$. The structures of the reduced protein with substrate bound show that $\mathrm{Gal}$ is properly positioned for interaction with the flavin $[99,101]$.

Much less is known about the mechanism and structure of eukaryotic UGMs. These enzymes share low sequence identity, and the presence of inserts in the primary structure predicts significant structural differences (Figure 6). In fact, comparison of the oligomeric states between prokaryotic and eukaryotic UGMs indicates that quaternary structures vary among species (Table 1) [93]. Furthermore, our group, as well as others, has demonstrated that known inhibitors of eukaryotic UGM are not effective or have decreased potency against L. major, A. fumigatus, and T. cruzi UGMs [7] (Qi and Sobrado unpublished results). Therefore, mechanistic and structural work is urgently needed on the eukaryotic enzymes.

3.3. UDP-Galactofuranose Transferases. UDP- $\alpha-G a l f$ is synthesized in the cytosol by UGM and is transported into the Golgi where it is attached to the LPG and GIPLs by galactofuranosyl transferases (GalfTs) [102]. Currently, all known linkages of Galf in T. cruzi and Leishmania spp. are in the $\beta$ anomer conformation. The most extensively studied GalfT is LPG-1 from L. major and L. donovani. Studies on LPG-1 have revealed that it is localized to the Golgi apparatus, where it adds the $\beta$-Gal $f$ to the core LPG structure [102, 103]. LPG-1 is a metal glycosyltranferase with typical conserved motifs including a cytoplasmic tail, a transmembrane domain, and a DXD metal-binding motif [104]. LPG-1 has been shown to only be responsible for the addition of Galf to LPG and to not play a role in the addition of Galf in the GIPLs [103, 105]. Mutants with the deletion of $l p g-1$ gene in both L. major and L. donovani show LPG-1 to be important for LPG formation. Due to the lack of LPG, the mutant strains with $l p g-1$ gene deleted in $L$. major display 


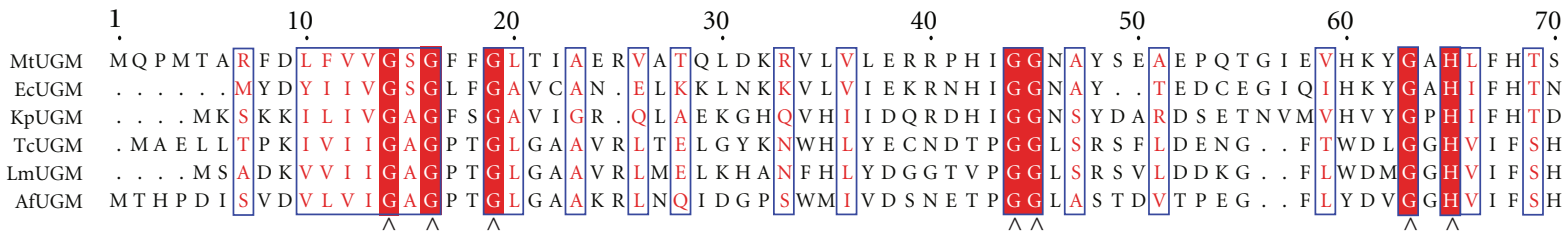

100

110

120

130

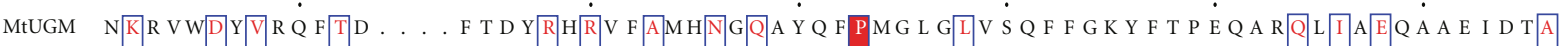

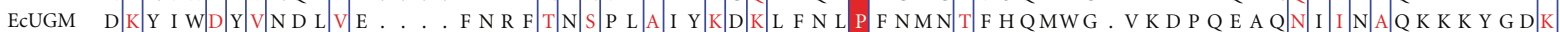
KpUGM NE T VWNY I N K HA E . . MMP Y V N R V K A T V N G Q V F S L P I N L H T I N Q F F S K T C S P D E A R A L I A E . K G D S T I A

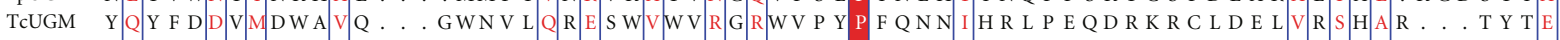
LmUGM Y Y Y F D D VMN L A I S ... DWN T L Q R E S S WV R C S G A A V P Y P F Q S N I H R L P P E V R D T C L K G I E E A E A A R S V A A P E AfUGM Y K Y F D D C L D E A L P K E D DWY T H Q R I S Y V R C C G Q WW V P Y P F Q N N I S M L P K E E Q V K C I D G M I D A A L E A R. V A N T

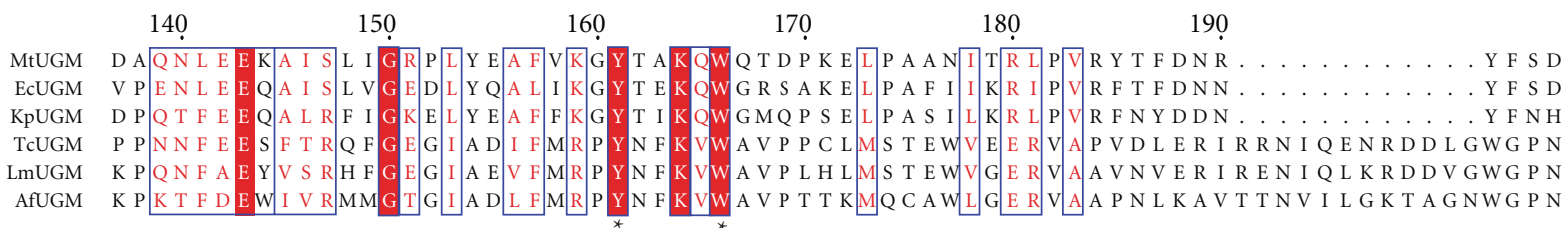

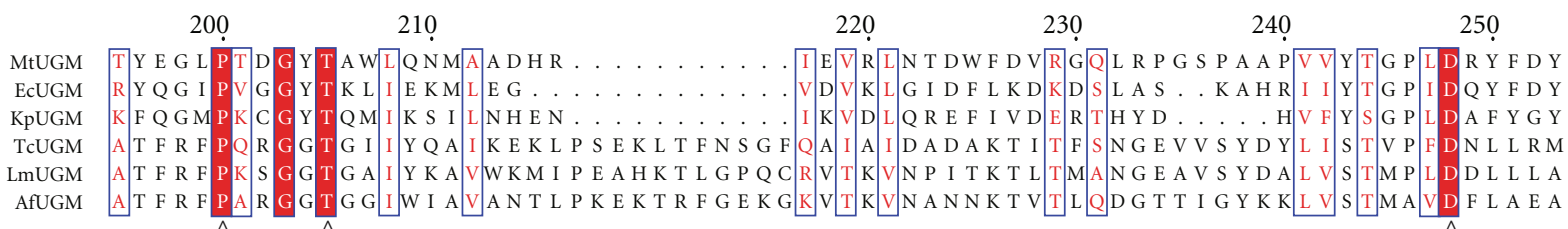

\begin{tabular}{rl|l}
\multicolumn{1}{c|}{260} \\
MtUGM
\end{tabular}

300

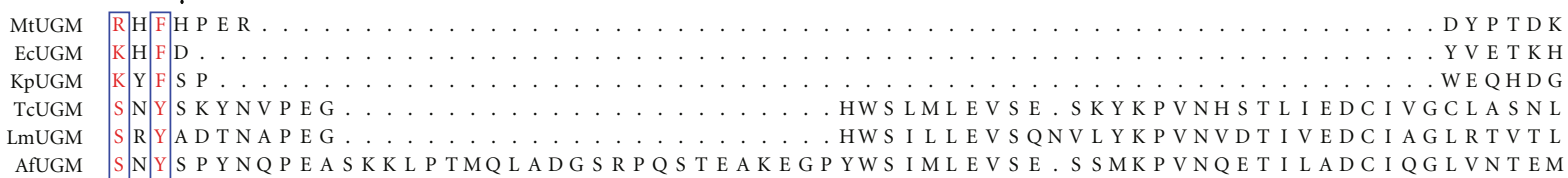

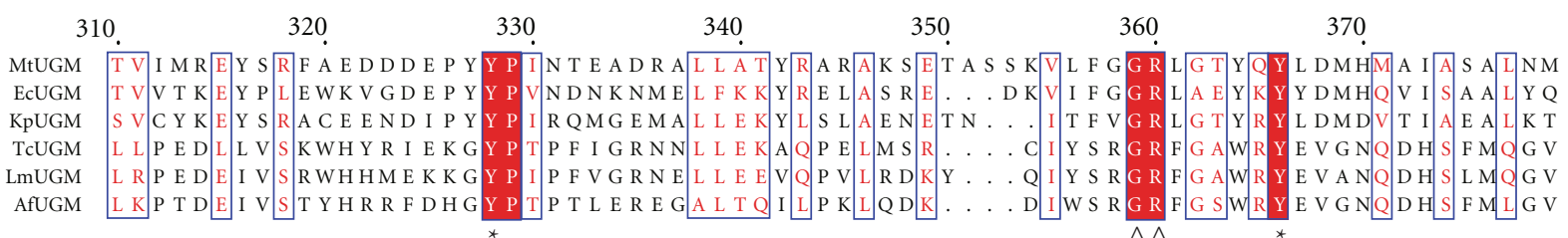

380

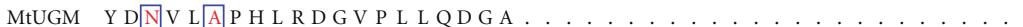

ECUGM V K N I M S T D D.

KPUGM A E V Y L N S L T D N Q PMP V F T V S V G

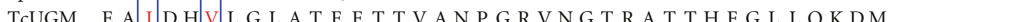

LmUGM E AVIGHIF. Y GT DE DTVHK P E KVNTRRGEMRCTWS S TA S . . . . .

AfUGM EAVDNLV. NGAVELTLNYPDFVNGRQNTERRLVDGAQVFAKS KA Q

FIGURE 6: Multiple sequence alignment of UDP-galactopyranose mutases. Conserved amino acids found in the active site of bacterial UGM are marked with a star, and those involved in flavin binding are marked with arrowheads. Mt: M. tuberculosis, Ec: E. coli; Kp, K. pneumoniae; Tc: T. cruzi; Lm: L. major; Af: A. fumigatus. The program ClustalW was used to generate the alignment and Espript 2.2 to create the figure [94]. 


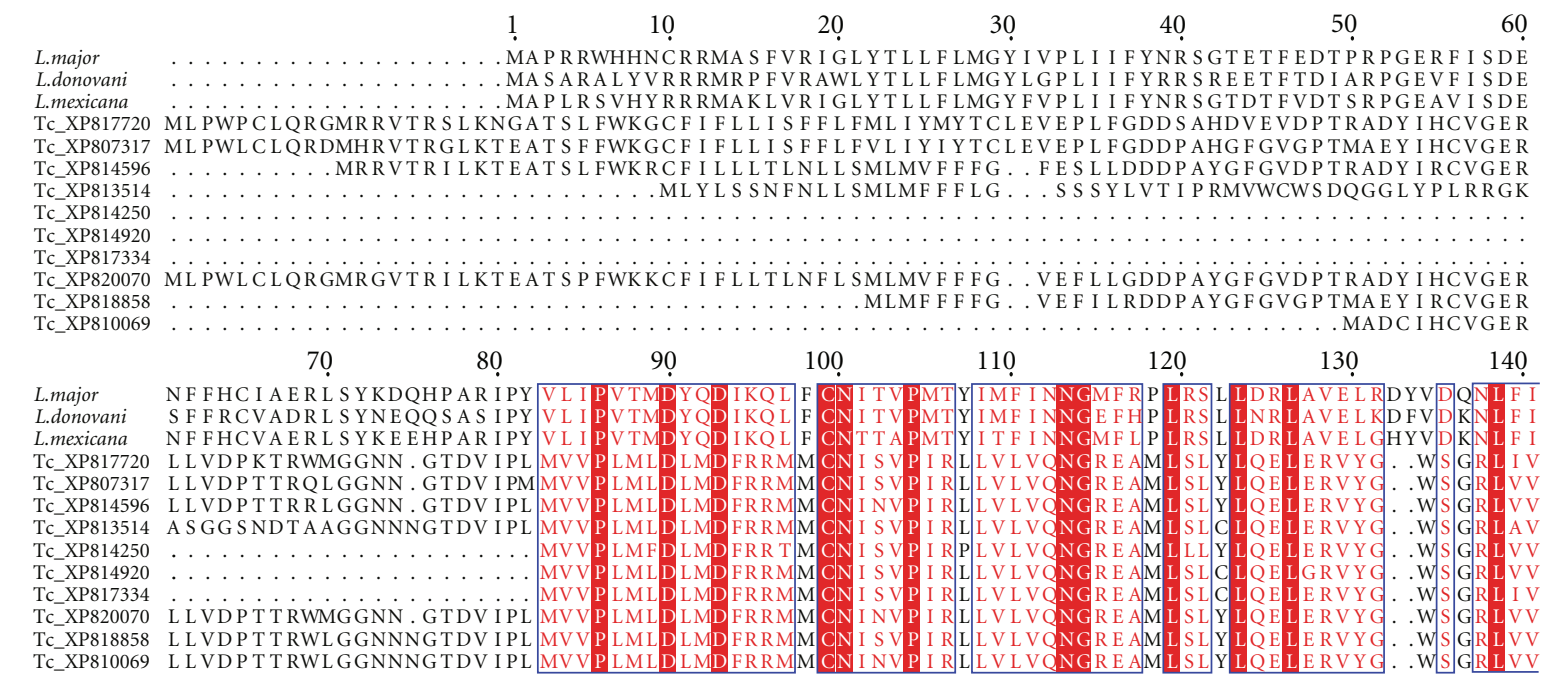
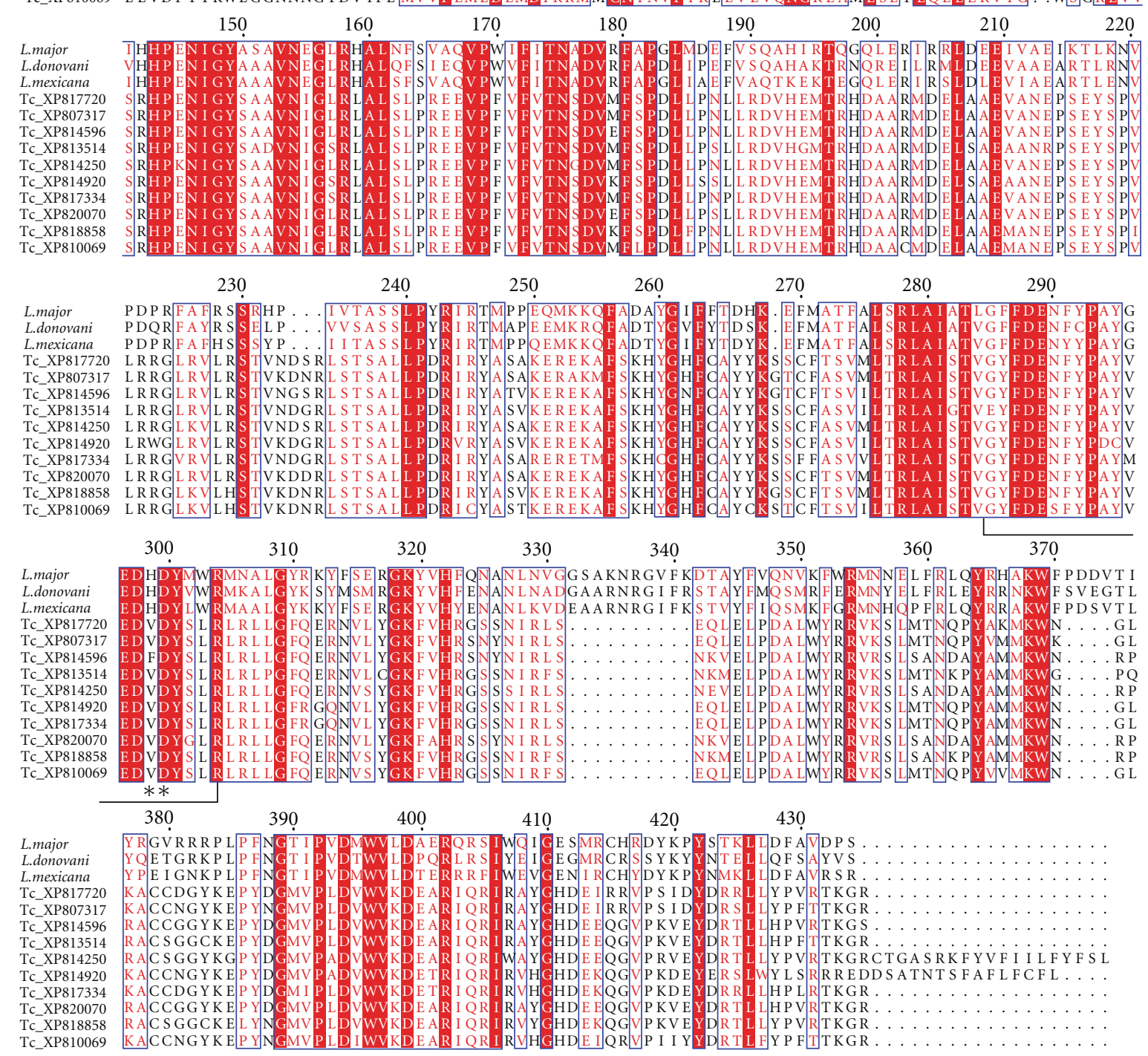

FIGURE 7: Alignment of L. major LPG-1 (XP001683753), L. donovani LPG-1 (ADG26596), L. mexicana LPG-1 (CAB6682), and ten putative T. cruzi GalfTs. Putative T. cruzi GalfTs were identified by BLAST search using L. major LPG-1 as the probe. The active site residues are shown in brackets, and the metal binding motif is represented with asterisks. The alignment was created as indicated in Figure 6. 
attenuated virulence $[103,105]$. These studies showed that LPG-1 could serve as a drug target in L. major.

There are no published studies on the GalfT from $T$. cruzi. In order to identify GalfTs in T. cruzi, a BLAST search was conducted using LPG-1 from $L$. major as a template, and more than 30 putative proteins annotated as $\beta$-GalfTs in the T. cruzi genome were identified [106, 107]. The top 10 putative GalfT sequences from the $T$. cruzi BLAST search were aligned with the $L$. major and $L$. donovani LPG-1 showing high sequence identity between these sequences (Figure 7). These sequences all contain the proposed catalytic site and demonstrate redundancy of the genes [104]. Redundancy of GalfTs is common in many different species, as often different transferases are used for each linkage type based on anomericity, bond linkage, and the substrate acceptors for Galf [108]. Due to the high number of GalfTs within T. cruzi, targeting GalfTs for drug design most likely would not be effective.

\section{Concluding Remarks}

To cause infection, protozoan parasites must recognize the mammalian host environment, bind and infect the target cells, and evade the immune system. Undoubtedly, the cell surface of these pathogens plays important roles in these processes. Current drugs are able to kill most of the parasites during treatment; however, these treatments do not eliminate all the parasites, presumably because they can "hide" in the intracellular forms. Modification of the cell surface sugar composition will alter the mechanism of infection. Enzymes involved in the biosynthesis of Galf have been shown to play a role in parasite growth and pathogenesis. GalE is essential for growth in T. cruzi and T. brucei, while UGM, and LPG-1 are important virulence factors in L. major [25, 82, 103]. Due to the presence of a GalE homolog in humans, compounds that inhibit this enzyme have toxic side effects. Furthermore, this enzyme is not important for virulence in Leishmania spp. UGM plays a central role in Galf biosynthesis and is the only source of UDP-Galf, which is the substrate for all the GalfT that attach Gal $f$ to the final sugar-acceptor molecules. Consequently, UGM emerges as an attractive drug candidate, as no homolog is found in humans [109]. The unique chemical structure of UGM suggests that specific inhibitors can be identified. Targeting UGM in T. cruzi and L. major will affect their virulence in humans and perhaps allow the immune system to effectively clear the parasite. Alternatively, inhibition of UGM will enhance the activity of other antiparasitic drugs. Such combination therapy might be necessary to combat these complex eukaryotic human pathogens.

\section{Acknowledgments}

This work was supported in part by NIH Grants RO1 GM094469 (P. Sobrado, PI) and RO1 AI082542 (R. Tarleton, PI). M. Oppenheimer was supported by a fellowship from the American Heart Association. A. L. Valenciano was supported by a fellowship from the Ministry of Science and Technology (MICIT) from Costa Rica.

\section{References}

[1] D. C. Crick, S. Mahapatra, and P. J. Brennan, "Biosynthesis of the arabinogalactan-peptidoglycan complex of Mycobacterium tuberculosis," Glycobiology, vol. 11, no. 9, 2001.

[2] G. Stevenson, B. Neal, D. Liu et al., "Structure of the O antigen of Escherichia coli K-12 and the sequence of its $\mathrm{rfb}$ gene cluster," Journal of Bacteriology, vol. 176, no. 13, pp. 4144-4156, 1994.

[3] M. Berst, C. G. Hellerqvist, B. Lindberg, O. Lüderitz, S. Svensson, and O. Westphal, "Structural investigations on T1 lipopolysaccharides," European Journal of Biochemistry, vol. 11, no. 2, pp. 353-359, 1969.

[4] C. Whitfield, J. C. Richards, M. B. Perry, B. R. Clarke, and L. L. MacLean, "Expression of two structurally distinct Dgalactan $\mathrm{O}$ antigens in the lipopolysaccharide of Klebsiella pneumoniae serotype O1," Journal of Bacteriology, vol. 173, no. 4, pp. 1420-1431, 1991.

[5] A. Weston, R. J. Stern, R. E. Lee et al., "Biosynthetic origin of mycobacterial cell wall galactofuranosyl residues," Tubercle and Lung Disease, vol. 78, no. 2, pp. 123-131, 1997.

[6] H. Bakker, B. Kleczka, R. Gerardy-Schahn, and F. H. Routier, "Identification and partial characterization of two eukaryotic UDP-galactopyranose mutases," Biological Chemistry, vol. 386, no. 7, pp. 657-661, 2005.

[7] S. M. Beverley, K. L. Owens, M. Showalter et al., "Eukaryotic UDP-galactopyranose mutase (GLF Gene) in microbial and metazoal pathogens," Eukaryotic Cell, vol. 4, no. 6, pp. 1147$1154,2005$.

[8] P. M. Nassau, S. L. Martin, R. E. Brown et al., "Galactofuranose biosynthesis in Escherichia coli K-12: identification and cloning of UDP-galactopyranose mutase," Journal of Bacteriology, vol. 178, no. 4, pp. 1047-1052, 1996.

[9] R. Köplin, J. R. Brisson, and C. Whitfield, "UDPgalactofuranose precursor required for formation of the lipopolysaccharide o antigen of Klebsiella pneumoniae serotype $\mathrm{O} 1$ is synthesized by the product of the $\mathrm{rfbD}(\mathrm{KPO} 1)$ gene," The Journal of Biological Chemistry, vol. 272, no. 7, pp. 4121-4128, 1997.

[10] M. Sarvas and H. Nikaido, "Biosynthesis of T1 antigen in Salmonella: origin of D-galactofuranose and D-ribofuranose residues," Journal of Bacteriology, vol. 105, no. 3, pp. 10631072, 1971.

[11] M. R. Richards and T. L. Lowary, "Chemistry and biology of galactofuranose-containing polysaccharides," ChemBioChem, vol. 10, no. 12, pp. 1920-1938, 2009.

[12] L. Kremer, L. G. Dover, C. Morehouse et al., "Galactan biosynthesis in Mycobacterium tuberculosis: identification of a bifunctional UDP-galactofuranosyltransferase," The Journal of Biological Chemistry, vol. 276, no. 28, pp. 26430-26440, 2001.

[13] F. Pan, M. Jackson, Y. Ma, and M. McNeil, "Cell wall core galactofuran synthesis is essential for growth of mycobacteria," Journal of Bacteriology, vol. 183, no. 13, pp. 3991-3998, 2001.

[14] E. C. Dykhuizen, J. F. May, A. Tongpenyai, and L. L. Kiessling, "Inhibitors of UDP-galactopyranose mutase thwart mycobacterial growth," Journal of the American Chemical Society, vol. 130, no. 21, pp. 6706-6707, 2008.

[15] E. C. Dykhuizen and L. L. Kiessling, "Potent ligands for prokaryotic UDP-galactopyranose mutase that exploit an enzyme subsite," Organic Letters, vol. 11, no. 1, pp. 193-196, 2009. 
[16] M. S. Scherman, K. A. Winans, R. J. Stern, V. Jones, C. R. Bertozzi, and M. R. McNeil, "Drug targeting Mycobacterium tuberculosis cell wall synthesis: development of a microtiter plate-based screen for UDP-galactopyranose mutase and identification of an inhibitor from a uridine-based library," Antimicrobial Agents and Chemotherapy, vol. 47, no. 1, pp. 378-382, 2003.

[17] M. Soltero-Higgin, E. E. Carlson, J. H. Phillips, and L. L. Kiessling, "Identification of inhibitors for UDPgalactopyranose mutase," Journal of the American Chemical Society, vol. 126, no. 34, pp. 10532-10533, 2004.

[18] J. P. Latge, "Galactofuranose containing molecules in Aspergillus fumigatus," Medical Mycology, vol. 47, supplement 1, pp. S104-S109, 2009.

[19] K. Barr, R. A. Laine, and R. L. Lester, "Carbohydrate structures of three novel phosphoinositol-containing sphingolipids from the yeast Histoplasma capsulatum," Biochemistry, vol. 23, no. 23, pp. 5589-5596, 1984.

[20] V. V. Vaishnav, B. E. Bacon, M. O'Neill, and R. Cherniak, "Structural characterization of the galactoxylomannan of Cryptococcus neoformans Cap67," Carbohydrate Research, vol. 306, no. 1-2, pp. 315-330, 1998.

[21] J. P. Latge, H. Kobayashi, J. P. Debeaupuis et al., "Chemical and immunological characterization of the extracellular galactomannan of Aspergillus fumigatus," Infection and Immunity, vol. 62, no. 12, pp. 5424-5433, 1994.

[22] M. Bernard and J. P. Latgé, "Aspergillus fumigatus cell wall: composition and biosynthesis," Medical Mycology, Supplement, vol. 39, supplement 1, pp. 9-17, 2001.

[23] P. S. Schmalhorst, S. Krappmann, W. Vervecken et al., "Contribution of galactofuranose to the virulence of the opportunistic pathogen Aspergillus fumigatus," Eukaryotic Cell, vol. 7, no. 8, pp. 1268-1277, 2008.

[24] J. Engel, P. S. Schmalhorst, T. Dörk-Bousset, V. Ferrières, and F. H. Routier, "A single UDP-galactofuranose transporter is required for galactofuranosylation in Aspergillus fumigatus," The Journal of Biological Chemistry, vol. 284, no. 49, pp. 33859-33868, 2009.

[25] B. Kleczka, A. C. Lamerz, G. van Zandbergen et al., "Targeted gene deletion of Leishmania major UDP-galactopyranose mutase leads to attenuated virulence," The Journal of Biological Chemistry, vol. 282, no. 14, pp. 10498-10505, 2007.

[26] R. M. de Lederkremer and W. Colli, "Galactofuranosecontaining glycoconjugates in trypanosomatids," Glycobiology, vol. 5, no. 6, pp. 547-552, 1995.

[27] R. M. de Lederkremer and R. Agusti, "Chapter 7 Glycobiology of Trypanosoma cruzi," Advances in Carbohydrate Chemistry and Biochemistry, vol. 62, pp. 311-366, 2009.

[28] J. R. Coura and J. Borges-Pereira, "Chagas disease: 100 years after its discovery. A systemic review," Acta Tropica, vol. 115, no. 1-2, pp. 5-13, 2010.

[29] W. De Souza, "Basic cell biology of Trypanosoma cruzi," Current Pharmaceutical Design, vol. 8, no. 4, pp. 269-285, 2002.

[30] H. Kato, E. A. Gomez, A. G. Cáceres, H. Uezato, T. Mimori, and Y. Hashiguchi, "Molecular epidemiology for vector research on leishmaniasis," International Journal of Environmental Research and Public Health, vol. 7, no. 3, pp. 814-826, 2010.

[31] J. A. Urbina, "Specific chemotherapy of Chagas disease: relevance, current limitations and new approaches," Acta Tropica, vol. 115, no. 1-2, pp. 55-68, 2010.
[32] L. Kedzierski, A. Sakthianandeswaren, J. M. Curtis, P. C. Andrews, P. C. Junk, and K. Kedzierska, "Leishmaniasis: current treatment and prospects for new drugs and vaccines," Current Medicinal Chemistry, vol. 16, no. 5, pp. 599-614, 2009.

[33] J. V. Richard and K. A. Werbovetz, "New antileishmanial candidates and lead compounds," Current Opinion in Chemical Biology, vol. 14, pp. 447-455, 2010.

[34] M. A. J. Ferguson, "The surface glycoconjugates of trypanosomatid parasites," Philosophical Transactions of the Royal Society B: Biological Sciences, vol. 352, no. 1359, pp. 1295-1302, 1997.

[35] S. J. Turco and A. Descoteaux, "The lipophosphoglycan of Leishmania parasites," Annual Review of Microbiology, vol. 46, pp. 65-94, 1992.

[36] D. B. Golgher, W. Colli, T. Souto-Padron, and B. Zingales, "Galactofuranose-containing glycoconjugates of epimastigote and trypomastigote forms of Trypanosoma cruzi," Molecular and Biochemical Parasitology, vol. 60, no. 2, pp. 249-264, 1993.

[37] P. A. Frey, "The Leloir pathway: a mechanistic imperative for three enzymes to change the stereochemical configuration of a single carbon in galactose," The FASEB Journal, vol. 10, no. 4, pp. 461-470, 1996.

[38] H. M. Holden, I. Rayment, and J. B. Thoden, "Structure and function of enzymes of the leloir pathway for galactose metabolism," The Journal of Biological Chemistry, vol. 278, no. 45, pp. 43885-43888, 2003.

[39] K. J. Isselbacher, "Evidence for an accessory pathway of galactose metabolism in mammalian liver," Science, vol. 126, no. 3275, pp. 652-654, 1957.

[40] S. J. Turco, M. A. Wilkerson, and D. R. Clawson, "Expression of an unusual acidic glycoconjugate in Leishmania donovani," The Journal of Biological Chemistry, vol. 259, no. 6, pp. 3883-3889, 1984.

[41] S. Damerow, A. C. Lamerz, T. Haselhorst et al., "Leishmania UDP-sugar pyrophosphorylase: the missing link in galactose salvage?" The Journal of Biological Chemistry, vol. 285, no. 2, pp. 878-887, 2010.

[42] A. Dickmanns, S. Damerow, P. Neumann et al., "Structural basis for the broad substrate range of the UDPsugar pyrophosphorylase from leishmania major," Journal of Molecular Biology, vol. 405, no. 2, pp. 461-478, 2011.

[43] A.-C. Lamerz, S. Damerow, B. Kleczka et al., "Deletion of UDP-glucose pyrophosphorylase reveals a UDP-glucose independent UDP-galactose salvage pathway in Leishmania major," Glycobiology, vol. 20, no. 7, pp. 872-882, 2010.

[44] R. Eisenthal, S. Game, and G. D. Holman, "Specificity and kinetics of hexose transport in Trypanosoma brucei," Biochimica et Biophysica Acta, vol. 985, no. 1, pp. 81-89, 1989.

[45] J. R. Roper, M. L. S. Güther, K. G. Milne, and M. A. J. Ferguson, "Galactose metabolism is essential for the african sleeping sickness parasite Trypanosoma brucei," Proceedings of the National Academy of Sciences of the United States of America, vol. 99, no. 9, pp. 5884-5889, 2002.

[46] M. A. Carver and S. J. Turco, "Cell-free biosynthesis of lipophosphoglycan from Leishmania donovani: characterization of microsomal galactosyltransferase and mannosyltransferase activities," The Journal of Biological Chemistry, vol. 266, no. 17, pp. 10974-10981, 1991.

[47] G. F. Späth, L. A. Garraway, S. J. Turco, and S. M. Beverley, "The role(s) of lipophosphoglycan (LPG) in the 
establishment of Leishmania major infections in mammalian hosts," Proceedings of the National Academy of Sciences of the United States of America, vol. 100, no. 16, pp. 9536-9541, 2003.

[48] S. J. Turco, G. F. Späth, and S. M. Beverley, "Is lipophosphoglycan a virulence factor? A surprising diversity between Leishmania species," Trends in Parasitology, vol. 17, no. 5, pp. 223-226, 2001.

[49] A. Svárovská, T. H. Ant, V. Seblová, L. Jecná, S. M. Beverley, and P. Volf, "Leishmania major glycosylation mutants require phosphoglycans $\left(p g 2^{-}\right)$but not lipophosphoglycan $\left(l l p g 1^{-}\right)$ for survival in permissive sand fly vectors," PLoS Neglected Tropical Diseases, vol. 4, no. 1, article e580, 2010.

[50] B. A. Butcher, S. J. Turco, B. A. Hilty, P. F. Pimentai, M. Panunzio, and D. L. Sacks, "Deficiency in $\beta 1,3-$ galactosyltransferase of a Leishmania major lipophosphoglycan mutant adversely influences the Leishmania-sand fly interaction," The Journal of Biological Chemistry, vol. 271, no. 34, pp. 20573-20579, 1996.

[51] M. J. McConville, S. W. Homans, J. E. Thomas-Oates, A. Dell, and A. Bacic, "Structures of the glycoinositolphospholipids from Leishmania major. A family of novel galactofuranosecontaining glycolipids," The Journal of Biological Chemistry, vol. 265, no. 13, pp. 7385-7394, 1990.

[52] R. M. de Lederkremer, O. L. Casal, M. J. Alves, and W. Colli, "Evidence for the presence of D-galactofuranose in the lipopeptidophosphoglycan from Trypanosome cruzi. Modification and tritium labeling," The FEBS Letters, vol. 116, no. 1, pp. 25-29, 1980.

[53] L. Mendonça-Previato, P. A. J. Gorin, A. F. Braga, J. Scharfstein, and J. O. Previato, "Chemical structure and antigenic aspects of complexes obtained from epimastigotes of Trypanosome cruzi," Biochemistry, vol. 22, no. 21, pp. 4980-4987, 1983.

[54] R. M. de Lederkremer, C. Lima, M. I. Ramirez, M. A. J. Ferguson, S. W. Homans, and J. Thomas-Oates, "Complete structure of the glycan of lipopeptidophosphoglycan from Trypanosome cruzi epimastigotes," The Journal of Biological Chemistry, vol. 266, no. 35, pp. 23670-23675, 1991.

[55] N. F. S. Nogueira, M. S. Gonzalez, J. E. Gomes et al., “Trypanosome cruzi: involvement of glycoinositolphospholipids in the attachment to the luminal midgut surface of Rhodnius prolixus," Experimental Parasitology, vol. 116, no. 2, pp. 120128, 2007.

[56] M. A. J. Ferguson, "The structure, biosynthesis and functions of glycosylphosphatidylinositol anchors, and the contributions of Trypanosome research," Journal of Cell Science, vol. 112, no. 17, pp. 2799-2809, 1999.

[57] E. Suzuki, A. K. Tanaka, M. S. Toledo, S. B. Levery, A. H. Straus, and H. K. Takahashi, "Trypanosomatid and fungal glycolipids and sphingolipids as infectivity factors and potential targets for development of new therapeutic strategies," Biochimica et Biophysica Acta, vol. 1780, no. 3, pp. 362-369, 2008.

[58] J. C. Carreira, C. Jones, R. Wait, J. O. Previato, and L. Mendonça-Previato, "Structural variation in the glycoinositolphospholipids of different strains of Trypanosome cruzi," Glycoconjugate Journal, vol. 13, no. 6, pp. 955-966, 1996.

[59] M. J. McConville and M. A. J. Ferguson, "The structure, biosynthesis and function of glycosylated phosphatidylinositols in the parasitic protozoa and higher eukaryotes," Biochemical Journal, vol. 294, no. 2, pp. 305-324, 1993.
[60] E. Suzuki, A. K. Tanaka, M. S. Toledo, H. K. Takahashi, and A. H. Straus, "Role of $\beta$-D-galactofuranose in Leishmania major macrophage invasion," Infection and Immunity, vol. 70, no. 12, pp. 6592-6596, 2002.

[61] R. M. de Lederkremer, C. E. Lima, M. I. Ramirez, M. F. Goncalvez, and W. Colli, "Hexadecylpalmitoylglycerol or ceramide is linked to similar glycophosphoinositol anchorlike structures in Trypanosome cruzi," European Journal of Biochemistry, vol. 218, no. 3, pp. 929-936, 1993.

[62] R. M. de Lederkremer, "Free and protein-linked glycoinositolphospholipids in Trypanosome cruzi," Brazilian Journal of Medical and Biological Research, vol. 27, no. 2, pp. 239-242, 1994.

[63] J. O. Previato, R. Wait, C. Jones et al., "Glycoinositolphospholipid from Trypanosome cruzi: structure, biosynthesis and immunobiology," Advances in Parasitology, vol. 56, pp. 1-41, 2003.

[64] J. I. MacRae, A. Acosta-Serrano, N. A. Morrice, A. Mehlert, and M. A. J. Ferguson, "Structural characterization of NETNES, a novel glycoconjugate in Trypanosome cruzi epimastigotes," The Journal of Biological Chemistry, vol. 280, no. 13, pp. 12201-12211, 2005.

[65] V. L. Pereira-Chioccola, A. Acosta-Serrano, I. C. de Almeida et al., "Mucin-like molecules form a negatively charged coat that protects Trypanosome cruzi trypomastigotes from killing by human anti- $\alpha$-galactosyl antibodies," Journal of Cell Science, vol. 113, no. 7, pp. 1299-1307, 2000.

[66] E. Suzuki, R. A. Mortara, H. K. Takahashi, and A. H. Straus, "Reactivity of MEST-1 (Antigalactofuranose) with Trypanosome cruzi glycosylinositol phosphorylceramides (GIPCs): immunolocalization of gipcs in acidic vesicles of epimastigotes," Clinical and Diagnostic Laboratory Immunology, vol. 8, no. 5, pp. 1031-1035, 2001.

[67] C. T. Moraes, M. Bosch, and A. J. Parodi, "Structural characterization of several galactofuranose-containing, highmannose-type oligosaccharides present in glycoproteins of the trypanosomatid leptomonas samueli," Biochemistry, vol. 27, no. 5, pp. 1543-1549, 1988.

[68] D. H. Mendelzon, J. O. Previato, and A. J. Parodi, "Characterization of protein-linked oligosaccharides in trypanosomatid flagellates," Molecular and Biochemical Parasitology, vol. 18, no. 3, pp. 355-367, 1986.

[69] D. H. Mendelzon and A. J. Parodi, "N-linked high mannosetype oligosaccharides in the protozoa Crithidia fasciculata and Crithidia harmosa contain galactofuranose residues," The Journal of Biological Chemistry, vol. 261, no. 5, pp. 21292133, 1986.

[70] D. C. Turnock and M. A. J. Ferguson, "Sugar nucleotide pools of Trypanosome brucei, Trypanosome cruzi, and Leishmania major," Eukaryotic Cell, vol. 6, no. 8, pp. 1450-1463, 2007.

[71] C. A. Buscaglia, V. A. Campo, A. C. Frasch, and J. M. Di Noia, "Trypanosome cruzi surface mucins: host-dependent coat diversity," Nature Reviews. Microbiology, vol. 4, no. 3, pp. 229-236, 2006.

[72] C. Jones, A. R. Todeschini, O. A. Agrellos, J. O. Previato, and L. Mendonça-Previato, "Heterogeneity in the biosynthesis of mucin O-glycans from Trypanosome cruzi Tulahuen strain with the expression of novel galactofuranosyl-containing oligosaccharides," Biochemistry, vol. 43, no. 37, pp. 1188911897, 2004.

[73] O. A. Agrellos, C. Jones, A. R. Todeschini, J. O. Previato, and L. Mendonça-Previato, "A novel sialylated and 
galactofuranose-containing O-linked glycan, Neu5Ac $\alpha 2 \rightarrow$ $3 \mathrm{Galp} \beta 1 \rightarrow 6(\mathrm{Galf} \beta 1 \rightarrow 4) \mathrm{GlcNAc}$, is expressed on the sialoglycoprotein of Trypanosome cruzi Dm28c," Molecular and Biochemical Parasitology, vol. 126, no. 1, pp. 93-96, 2003.

[74] J. O. Previato, C. Jones, L. P. B. Goncalves, R. Wait, L. R. Travassos, and L. Mendonca-Previato, "O-Glycosidically linked $\mathrm{N}$-acetylglucosamine-bound oligosaccharides from glycoproteins of Trypanosome cruzi," Biochemical Journal, vol. 301, no. 1, pp. 151-159, 1994.

[75] A. A. Serrano, S. Schenkman, N. Yoshida, A. Mehlert, J. M. Richardson, and M. A. J. Ferguson, "The lipid structure of the glycosylphosphatidylinositol-anchored mucin- like sialic acid acceptors of Trypanosome cruzi changes during parasite differentiation from epimastigotes to infective metacyclic trypomastigote forms," The Journal of Biological Chemistry, vol. 270, no. 45, pp. 27244-27253, 1995.

[76] I. C. Almeida, M. A. J. Ferguson, S. Schenkman, and L. R. Travassos, "Lytic anti- $\alpha$-galactosyl antibodies from patients with chronic Chagas' disease recognize novel O-linked oligosaccharides on mucin-like glycosyl-phosphatidylinositol-anchored glycoproteins of Trypanosome cruzi," Biochemical Journal, vol. 304, no. 3, pp. 793-802, 1994.

[77] A. Acosta-Serrano, I. C. Almeida, L. H. Freitas-Junior, N. Yoshida, and S. Schenkman, "The mucin-like glycoprotein super-family of Trypanosome cruzi: structure and biological roles," Molecular and Biochemical Parasitology, vol. 114, no. 2, pp. 143-150, 2001.

[78] A. R. Todeschini, E. X. da Silveira, C. Jones, R. Wait, J. O. Previato, and L. Mendonça-Previato, "Structure of O-glycosidically linked oligosaccharides from glycoproteins of Trypanosome cruzi CL-Brener strain: evidence for the presence of o-linked sialyl-oligosaccharides," Glycobiology, vol. 11, no. 1, pp. 47-55, 2001.

[79] J. O. Previato, C. Jones, M. T. Xavier et al., "Structural characterization of the major glycosylphosphatidylinositol membrane-anchored glycoprotein from epimastigote forms of Trypanosome cruzi Y-strain," The Journal of Biological Chemistry, vol. 270, no. 13, pp. 7241-7250, 1995.

[80] E. Suzuki, M. S. Toledo, H. K. Takahashi, and A. H. Straus, "A monodonal antibody directed to terminal residue of $\beta$ galactofuranose of a glycolipid antigen isolated from Paracoccidioides brasiliensis: cross-reactivity with Leishmania major and Trypanosome cruzi," Glycobiology, vol. 7, no. 4, pp. 463-468, 1997.

[81] M. V. De Arruda, W. Colli, and B. Zingales, “Terminal $\beta$ D-galactofuranosyl epitopes recognized by antibodies that inhibit Trypanosome cruzi internalization into mammalian cells," European Journal of Biochemistry, vol. 182, no. 2, pp. 413-421, 1989.

[82] J. I. MacRae, S. O. Obado, D. C. Turnock et al., "The suppression of galactose metabolism in Trypanosome cruzi epimastigotes causes changes in cell surface molecular architecture and cell morphology," Molecular and Biochemical Parasitology, vol. 147, no. 1, pp. 126-136, 2006.

[83] U. Oppermann, C. Filling, M. Hult et al., "Short-chain dehydrogenases/reductases (SDR): the 2002 update," ChemicoBiological Interactions, vol. 143-144, pp. 247-253, 2003.

[84] M. P. Shaw, C. S. Bond, J. R. Roper, D. G. Gourley, M. A. J. Ferguson, and W. N. Hunter, "High-resolution crystal structure of Trypanosome brucei UDP-galactose 4'-epimerase: a potential target for structure-based development of novel trypanocides," Molecular and Biochemical Parasitology, vol. 126, no. 2, pp. 173-180, 2003.
[85] M. S. Alphey, A. Burton, M. D. Urbaniak, G. J. Boons, M. A. J. Ferguson, and W. N. Hunter, "Trypanosome brucei UDPgalactose-4'-epimerase in ternary complex with NAD and the substrate analogue UDP-4-deoxy-4-fluoro- $\alpha$-D- galactose," Acta Crystallographica Section F: Structural Biology and Crystallization Communications, vol. 62, no. 9, pp. 829-834, 2006.

[86] J. R. Roper, M. L. S. Güther, J. I. MacRae et al., "The suppression of galactose metabolism in procylic form Trypanosome brucei causes cessation of cell growth and alters procyclin glycoprotein structure and copy number," The Journal of Biological Chemistry, vol. 280, no. 20, pp. 19728-19736, 2005.

[87] M. D. Urbaniak, D. C. Turnock, and M. A. J. Ferguson, "Galactose starvation in a bloodstream form Trypanosome brucei UDP-glucose 4'-epimerase conditional null mutant," Eukaryotic Cell, vol. 5, no. 11, pp. 1906-1913, 2006.

[88] R. J. S. Burchmore, D. Rodriguez-Contrerast, K. McBride et al., "Genetic characterization of glucose transporter function in Leishmania mexicana," Proceedings of the National Academy of Sciences of the United States of America, vol. 100, no. 7, pp. 3901-3906, 2003.

[89] M. D. Urbaniak, J. N. Tabudravu, A. Msaki et al., "Identification of novel inhibitors of UDP-Glc $4 /$-epimerase, a validated drug target for african sleeping sickness," Bioorganic and Medicinal Chemistry Letters, vol. 16, no. 22, pp. 57445747, 2006.

[90] J. D. Durrant, M. D. Urbaniak, M. A. J. Ferguson, and J. A. McCammon, "Computer-aided identification of Trypanosome brucei uridine diphosphate galactose 4 '-epimerase inhibitors: toward the development of novel therapies for African sleeping sickness," Journal of Medicinal Chemistry, vol. 53, no. 13, pp. 5025-5032, 2010.

[91] D. A. R. Sanders, S. A. McMahon, G. L. Leonard, and J. H. Naismith, "Molecular placement of experimental electron density: a case study on UDP-galactopyranose mutase," Acta Crystallographica-Section D Biological Crystallography, vol. 57, no. 10, pp. 1415-1420, 2001.

[92] K. Beis, V. Srikannathasan, H. Liu et al., "Crystal structures of Mycobacteria tuberculosis and Klebsiella pneumoniae UDPgalactopyranose Mutase in the oxidised state and Klebsiella pneumoniae UDP-galactopyranose mutase in the (active) reduced state," Journal of Molecular Biology, vol. 348, no. 4, pp. 971-982, 2005.

[93] M. Oppenheimer, M. B. Poulin, T. L. Lowary, R. F. Helm, and P. Sobrado, "Characterization of recombinant UDP-galactopyranose mutase from Aspergillus fumigatus," Archives of Biochemistry and Biophysics, vol. 502, no. 1, pp. 31-38, 2010.

[94] P. Gouet, E. Courcelle, D. I. Stuart, and F. Métoz, "ESPript: analysis of multiple sequence alignments in PostScript," Bioinformatics, vol. 15, no. 4, pp. 305-308, 1999.

[95] A. M. El-Ganiny, D. A. R. Sanders, and S. G. W. Kaminskyj, "Aspergillus nidulans UDP-galactopyranose mutase, encoded by ugmA plays key roles in colony growth, hyphal morphogensis, and conidiation," Fungal Genetics and Biology, vol. 45, no. 12, pp. 1533-1542, 2008.

[96] Q. Zhang and H. W. Liu, "Studies of UDP-galactopyranose mutase from Escherichia coli: an unusual role of reduced FAD in its catalysis," Journal of the American Chemical Society, vol. 122, no. 38, pp. 9065-9070, 2000.

[97] D. A. R. Sanders, A. G. Staines, S. A. McMahon, M. R. McNeil, C. Whitfield, and J. H. Naismith, "UDP-galactopyranose mutase has a novel structure and mechanism," Nature Structural Biology, vol. 8, no. 10, pp. 858-863, 2001. 
[98] M. Soltero-Higgin, E. E. Carlson, T. D. Gruber, and L. L. Kiessling, "A unique catalytic mechanism for UDPgalactopyranose mutase," Nature Structural and Molecular Biology, vol. 11, no. 6, pp. 539-543, 2004.

[99] T. D. Gruber, W. M. Westler, L. L. Kiessling, and K. T. Forest, "X-Ray crystallography reveals a reduced substrate complex of UDP-galactopyranose mutase poised for covalent catalysis by flavin," Biochemistry, vol. 48, no. 39, pp. 9171-9173, 2009.

[100] Z. Huang, Q. Zhang, and H. W. Liu, "Reconstitution of UDPgalactopyranose mutase with 1-deaza-FAD and 5-deazaFAD: analysis and mechanistic implications," Bioorganic Chemistry, vol. 31, no. 6, pp. 494-502, 2003.

[101] S. Karunan Partha, K. E. van Straaten, and D. A. R. Sanders, "Structural basis of substrate binding to UDPgalactopyranose mutase: crystal structures in the reduced and oxidized state complexed with UDP-galactopyranose and UDP," Journal of Molecular Biology, vol. 394, no. 5, pp. 864-877, 2009.

[102] D. S. Ha, J. K. Schwarz, S. J. Turco, and S. M. Beverley, "Use of the green fluorescent protein as a marker in transfected Leishmania," Molecular and Biochemical Parasitology, vol. 77, no. 1, pp. 57-64, 1996.

[103] G. F. Späth, L. Epstein, B. Leader et al., "Lipophosphoglycan is a virulence factor distinct from related glycoconjugates in the protozoan parasite Leishmania major," Proceedings of the National Academy of Sciences of the United States of America, vol. 97, no. 16, pp. 9258-9263, 2000.

[104] K. Zhang, T. Barron, S. J. Turco, and S. M. Beverley, "The LPG1 gene family of Leishmania major," Molecular and Biochemical Parasitology, vol. 136, no. 1, pp. 11-23, 2004.

[105] K. A. Ryan, L. A. Garraway, A. Descoteaux, S. J. Turco, and S. M. Beverley, "Isolation of virulence genes directing surface glycosyl- phosphatidylinositol synthesis by functional complementation of Leishmania," Proceedings of the National Academy of Sciences of the United States of America, vol. 90, no. 18, pp. 8609-8613, 1993.

[106] N. M. El-Sayed, P. J. Myler, D. C. Bartholomeu et al., "The genome sequence of Trypanosoma cruzi, etiologic agent of chagas disease," Science, vol. 309, no. 5733, pp. 409-415+435, 2005.

[107] N. M. El-Sayed, P. J. Myler, G. Blandin et al., "Comparative genomics of trypanosomatid parasitic protozoa," Science, vol. 309, no. 5733, pp. 404-409+435, 2005.

[108] D. Kapitonov and R. K. Yu, "Conserved domains of glycosyltransferases," Glycobiology, vol. 9, no. 10, pp. 961-978, 1999.

[109] L. L. Pedersen and S. J. Turco, "Galactofuranose metabolism: a potential target for antimicrobial chemotherapy," Cellular and Molecular Life Sciences, vol. 60, no. 2, pp. 259-266, 2003. 

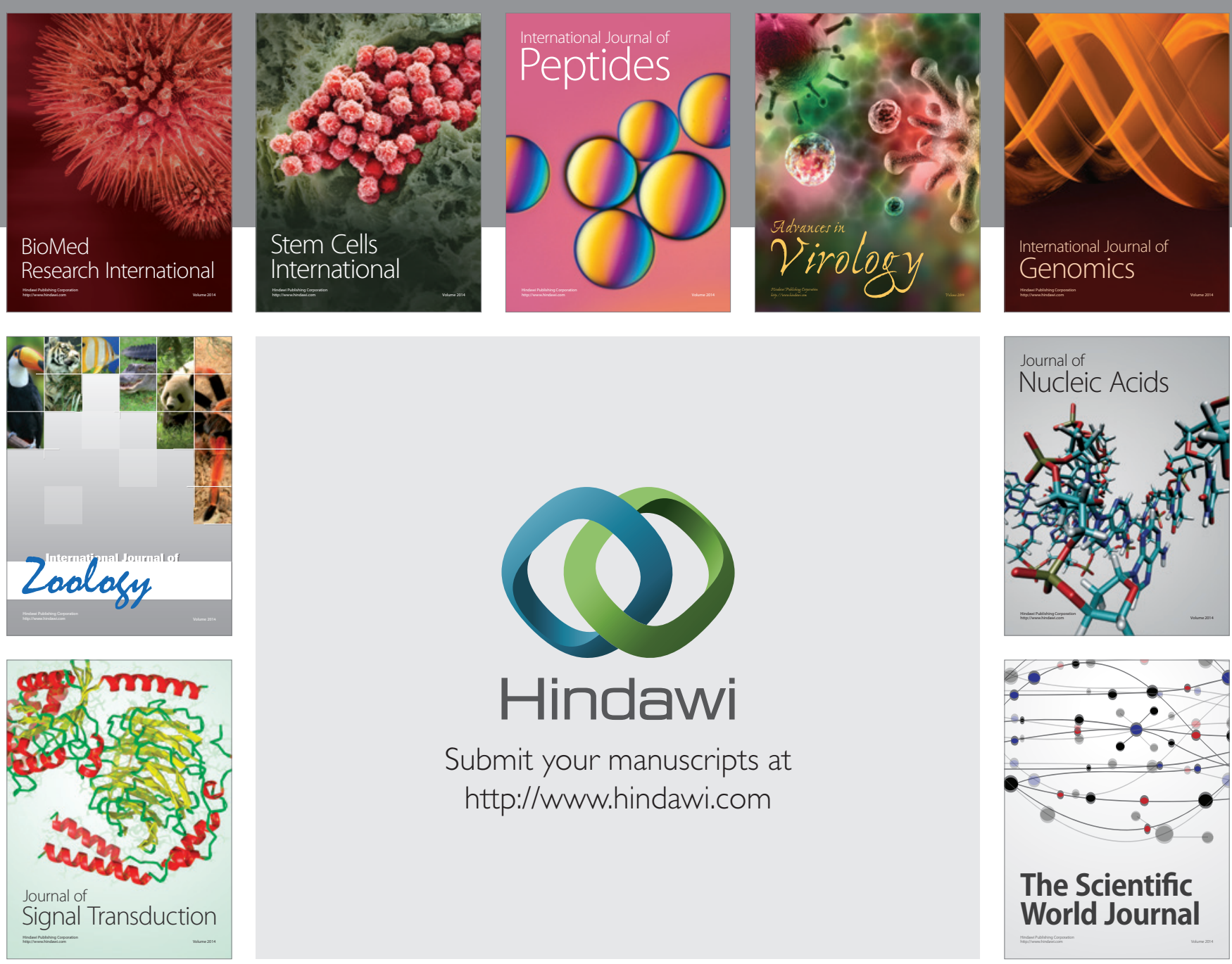

Submit your manuscripts at

http://www.hindawi.com
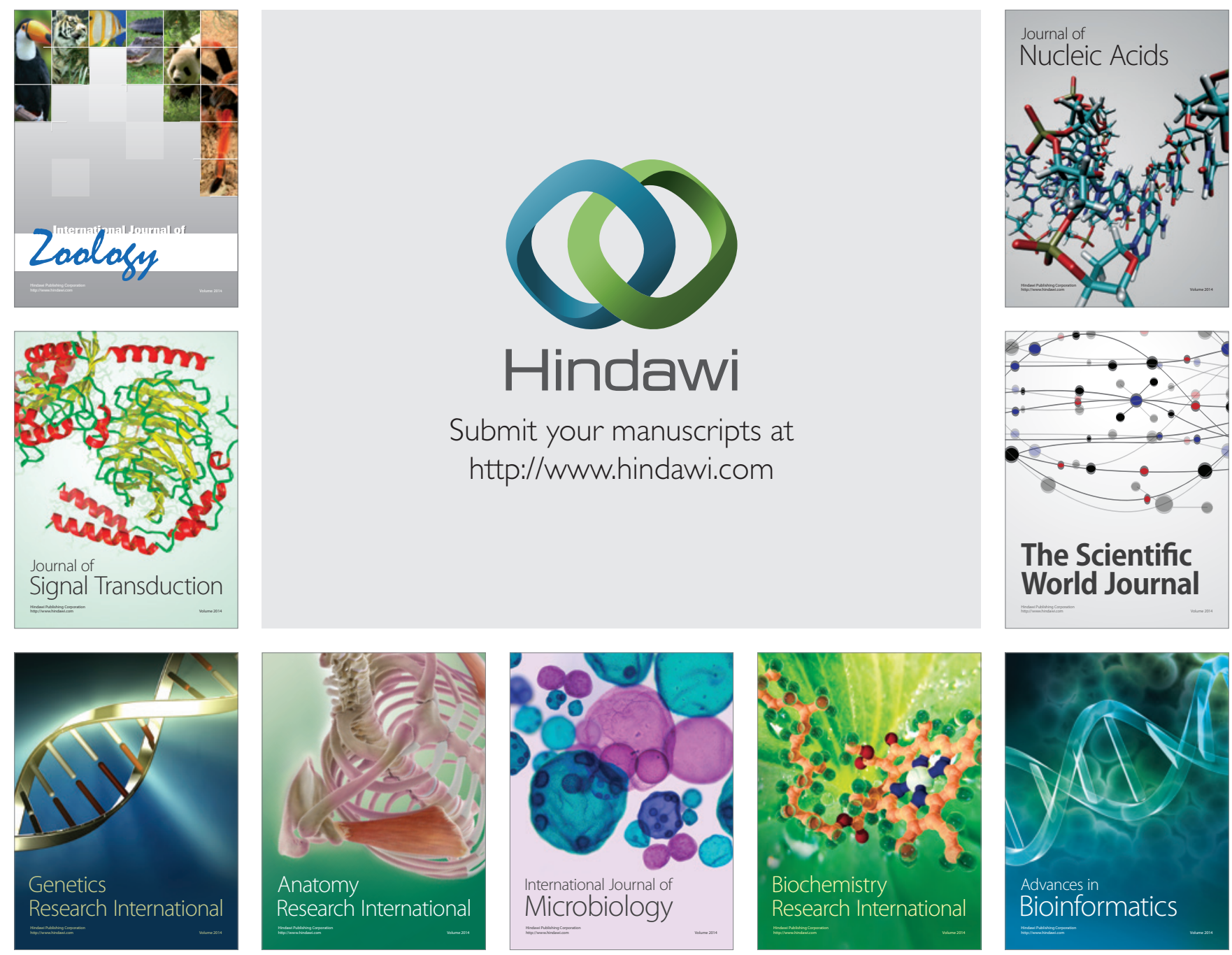

The Scientific World Journal
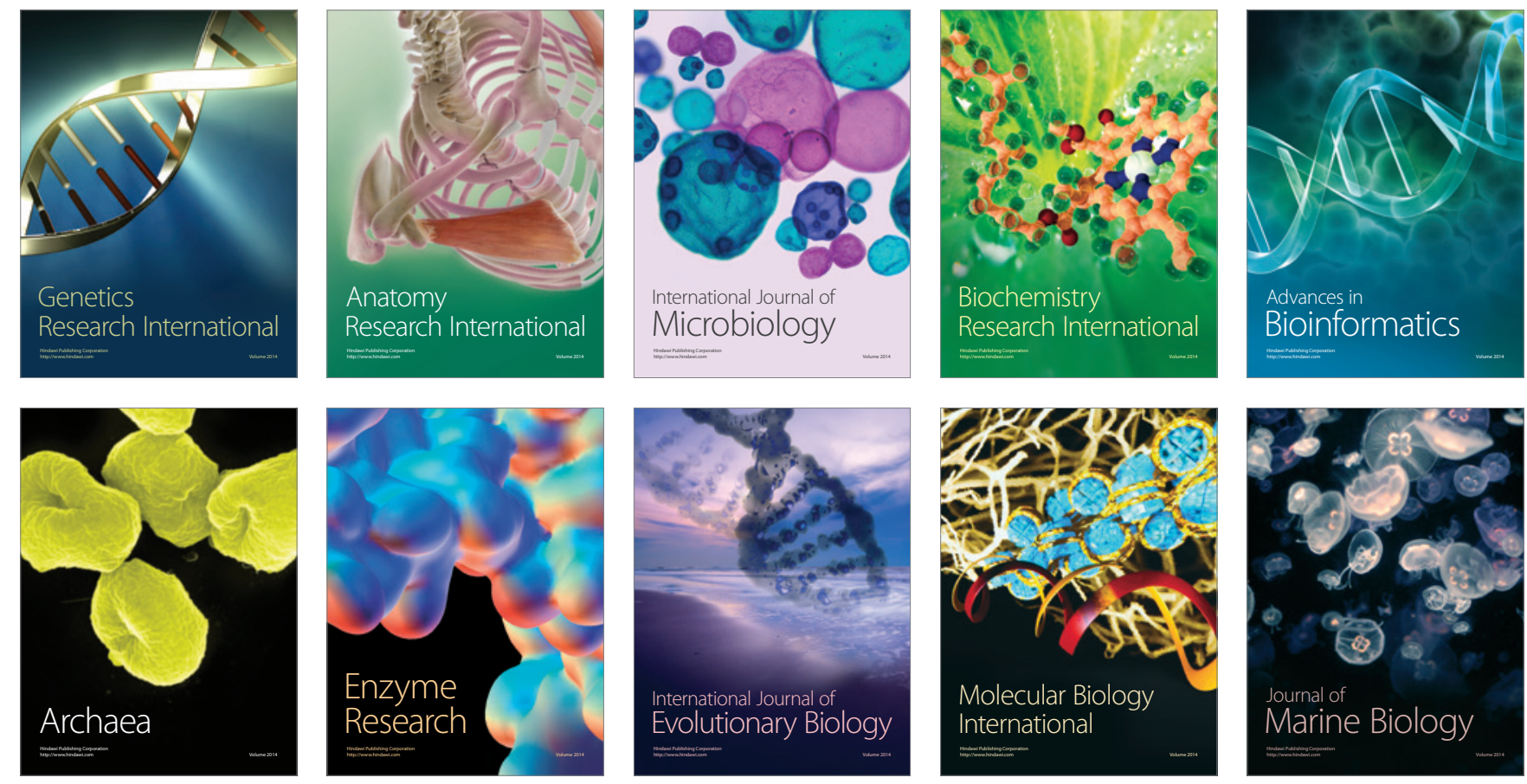